Commentary/O’Regan \& Noë: A sensorimotor account of vision and visual consciousness

\title{
Neural correlates of consciousness are not pictorial representations
}

\section{Geraint Rees ${ }^{\mathrm{a}}$ and Chris Frith ${ }^{\mathrm{b}}$}

a Institute of Cognitive Neuroscience, University College London, London WC1N 3AR, United Kingdom; ${ }^{\mathrm{b}}$ Wellcome Department of Cognitive

Neurology, University College London, London WC1N 3BG, United Kingdom. g.rees@fil.ion.ucl.ac.uk http://www.fil.ion.ucl.ac.uk/ grees c.frith@fil.ion.ucl.ac.uk http://www.fil.ion.ucl.ac.uk/principals.html

Abstract: O'Regan \& Noë $(\mathrm{O} \& \mathrm{~N})$ are pessimistic about the prospects for discovering the neural correlates of consciousness. They argue that there can be no one-to-one correspondence between awareness and patterns of neural activity in the brain, so a project attempting to identify the neural correlates of consciousness is doomed to failure. We believe that this degree of pessimism may be overstated; recent empirical data show some convergence in describing consistent patterns of neural activity associated with visual consciousness 
Commentary/O’Regan \& Noë: A sensorimotor account of vision and visual consciousness

A consistent finding in humans and monkeys is that damage to functionally specialized regions of the visual cortex leads to deficient awareness of the visual attribute represented in that area, without impairment of awareness for other attributes. For example, damage to the human homologue of V5/MT (middle temporal area) leads to akinetopsia (Zihl et al. 1983). Alteration of activity in that area through microstimulation (Salzman et al. 1990) or transcranial magnetic stimulation (Beckers \& Homberg 1992; though see Pascual-Leone \& Walsh 2001) leads to altered perception of that attribute. Even when sensory input and motor output are held constant, changes in perceptual awareness (Tong et al. 1998) or hallucinations (ffytche et al. 1998; Silbersweig et al. 1995) are associated with enhanced activity in areas of visual cortex with functional specializations that correspond to the percept experienced. These remarkably consistent results from a wide variety of experimental techniques suggest that activity in functionally specialized areas of visual cortex is correlated with, and necessary for, visual awareness.

We do not claim that this neural correlate of consciousness is constitutive of, or even sufficient for, visual awareness; merely that it represents a consistent (and probably necessary) correlate of consciousness. Recent neuroimaging experiments suggest that visual cortex activity can be detected for unseen stimuli in parietal extinction (Rees et al. 2000; Vuilleumier et al. 2001), during change blindness (Beck et al. 2001) or following pattern masking (Dehaene et al. 2001). When stimuli reach awareness, not only is enhanced activity observed in the ventral visual pathway, but also in areas of frontal and parietal cortex (Kleinschmidt et al. 1998; Lumer 2000). For the parietal cortex in particular, a remarkably consistent locus of activation correlated with awareness is seen in the superior parietal lobule (Rees 2001). Parietal and prefrontal cortices are often associated with attention and motor control, so the consistent involvement of areas associated with action in conscious awareness is supportive of the general notion of a close relationship between (conscious) perception and action. However, activity in these areas is correlated with awareness even when sensory stimulation and motor behavior is held constant (Frith et al. 1999).

A consistent association of parietal and prefrontal activity with visual awareness supports the notion that motor control systems may play an important role in visual awareness, as O'Regan \& Noë $(\mathbf{O \& N})$ suggest. However, it also implies that involvement of these systems can be independent of overt behavior (and consequent changes in the environment). Thus, an important qualification for their theoretical claims is that "mastery of sensorimotor contingencies" may reflect covert rather than overt behavior. The challenge for O\&N is therefore to specify how this covert "mastery of sensorimotor contingency" differs from other cognitive processes such as attention or working memory. Indeed, the notion that motor activity is not necessary for awareness is consistent with clinical observations. Damage to motor cortex leading to paralysis causes little or no change in conscious experience, either acutely or in the long term. Indeed, an individual almost completely paralysed (apart from the ability to blink) authored a bestselling volume of short stories, displaying apparently normal consciousness (Bauby 1997). This failure to identify changes in conscious experience following changes in the ability of individuals to master sensorimotor contingencies must place important constraints on O\&N's theoretical approach.

The notion of the neural correlates of visual consciousness as reflected in activity in a distributed network of cortical areas is rather different to $\mathbf{O \& N}$ 's characterization of such research as searching for a pictorial representation of the outside world inside the brain. No such representation is either assumed or implied by the research we review here. However, the consistent activation of specific areas poses a challenge to O\&N's predictions that no one-to-one correspondence between attributes represented in consciousness and brain activity is possible. We are sympathetic to the notion that consideration of action may have an important role to play in explaining how activity in the brain is associated with consciousness (Neisser 1967). However, empirical data does not support the wider implications of O\&N's claim that the study of the neural correlates of consciousness is doomed to inconsistency and failure.

\section{ACKNOWLEDGMENT}

The Wellcome Trust funded this work. 